\title{
Extending shear-wave tomography for the lower mantle using $S$ and SKS arrival-time data
}

\author{
S. Widiyantoro ${ }^{1,2}$, B. L. N. Kennett ${ }^{1}$, and R. D. van der Hilst $^{3}$ \\ ${ }^{1}$ Research School of Earth Sciences, The Australian National University, Canberra ACT 0200, Australia \\ ${ }^{2}$ Department of Geophysics and Meteorology, Bandung Institute of Technology, Bandung 40132, Indonesia \\ ${ }^{3}$ Department of Earth, Atmospheric and Planetary Sciences, Massachusetts Institute of Technology, Rm 54-514, Cambridge MA 02139, U.S.A.
}

(Received April 30, 1998; Revised August 10, 1998; Accepted August 20, 1998)

Seismic tomography using $\mathrm{S}$ wave travel times faces the difficulty imposed by the interference between $\mathrm{S}$ and SKS phases near $83^{\circ}$ epicentral distance, as the SKS phase overtakes the $S$ waves in the mantle. If the cross-over is avoided completely by excluding $\mathrm{S}$ data beyond $82^{\circ}$ then no resolution is available below $2200 \mathrm{~km}$ in the lower mantle. A partial solution is to try to pick up the $\mathrm{S}$ phase beyond the cross-over which improves coverage and resolution in depth. However, a much larger improvement can be made by following the first arrival with $\mathrm{S}$ character and including SKS information with S.

Arrival times for both S and SKS phases and the event hypocentres have been taken from the reprocessing of data reported to international agencies. Each event has been relocated, including depth phase information, and later phases re-associated using the improved locations to provide a set of travel times whose variance is significantly reduced compared with the original data catalogues.

$\mathrm{S}$ travel-time tomography including SKS information out to $105^{\circ}$, provides tomographic images with improved rendition of heterogeneity in the lower mantle. The three-dimensional models of SV wavespeed relative to the $a k 135$ reference velocity model show a significant increase in heterogeneity at the base of the mantle which matches the behaviour seen in results derived from waveform inversion.

For most of the mantle there is a considerable similarity between the patterns of heterogeneity in the $S$ wave images and recent $\mathrm{P}$ wave tomographic results, but greater differences develop in the lowermost mantle. In the D" region the SV wavespeed patterns also show some differences from recent SH wavespeed results which mostly correlate with regions of recognised structural complexity.

\section{Introduction}

Long period $\mathrm{S}$ wave information derived from analysis of digital records for both absolute and differential times has been incorporated into recent tomographic inversions using surface wave and free-oscillation data e.g. Masters et al. (1996). However such models have concentrated on the large features of the three-dimensional structure with a spherical harmonic analysis up to order 16. Finer resolution results for SH wave structure have been presented by Grand (1994) derived from picks from digital records around the globe for $\mathrm{S}$, $\mathrm{ScS}$ and their multiples and this work has now been extended to global coverage (Grand et al., 1997).

Whereas $\mathrm{P}$ wave tomography using travel-time data has been able to provide relatively detailed images of threedimensional structure for the whole mantle (see e.g. van der Hilst et al., 1997), there has been much less progress in Swave imaging using the comparable travel-time information reported to international agencies. Vasco et al. (1994) use a relatively coarse parametrisation for both $\mathrm{P}$ and $\mathrm{S}$ wave images, but $\mathrm{S}$ results are truncated at $2270 \mathrm{~km}$. More recently Robertson and Woodhouse $(1995,1996)$ have also used the ISC database to develop an $\mathrm{S}$ wave representation in terms

Copy right $(\mathrm{C}$ The Society of Geomagnetism and Earth, Planetary and Space Sciences (SGEPSS); The Seismological Society of Japan; The Volcanological Society of Japan; The Geodetic Society of Japan; The Japanese Society for Planetary Sciences. of spherical harmonics to angular order 12. Their S model initially extended to $2100 \mathrm{~km}$ and was subsequently extended deeper into the lower mantle following further processing of the $\mathrm{S}$ wave times. Kennett et al. (1998) have exploited both $\mathrm{P}$ and $\mathrm{S}$ arrival times for events with common source and station pairs in a joint inversion for three-dimensional bulksound and shear wavespeed structure in the mantle using a $2^{\circ} \times 2^{\circ}$ cellular representation.

The difficulty with $S$ wave imaging of structure in the lower mantle arises from the interference between the $S$ phase itself and the SKS arrival which has S character in the mantle and a $\mathrm{P}$ wave leg in the core. Because the $\mathrm{P}$ wavespeed at the top of the outer core exceeds the $S$ wavespeed at the base of the mantle SKS waves can travel faster than S. The crossover between $\mathrm{S}$ and SKS as the earlier arrival occurs close to $83^{\circ}$ for a shallow focus event. If attention is therefore to be restricted to $\mathrm{S}$ waves refracted back from the lower mantle there are two choices:

(i) to avoid the cross-over completely by using $\mathrm{S}$ travel times to $82^{\circ}$ which gives a maximum turning depth in the mantle of about $2100 \mathrm{~km}$.

(ii) to include also $\mathrm{S}$ waves beyond the cross-over (cf. Robertson and Woodhouse, 1996) which requires careful separation of S and SKS beyond $84^{\circ}$ and which now allows $S$ rays to penetrate most of the lower mantle.

The alternative, which we adopt here, is to make use of 
the information contained in the SKS data on the S structure in the mantle. We use $\mathrm{S}$ wave arrival-time data for distances out to $105^{\circ}$ and SKS data from $84^{\circ}$ to $105^{\circ}$.

Similar problems beset the construction of reference models for the radial distribution of $\mathrm{S}$, and the recent reference models derived from travel-time observations sp6 (Morelli and Dziewonski, 1993), ak135 (Kennett et al., 1995) have combined $\mathrm{S}$ and $\mathrm{S}$ core phase information in order to develop an $\mathrm{S}$ structure for the full mantle.

SKS beyond $84^{\circ}$ has the advantage that it is the first arriving phase with $\mathrm{S}$ character, in consequence it is both well reported and, since it attains its largest amplitude on horizontal components, emerges clearly from the coda of P. On the other hand beyond $84^{\circ}$ the $\mathrm{S}$ arrival has to be sought in the coda of SKS. We have restricted attention to SKS phases for distances in the range $84^{\circ}-105^{\circ}$ for which there is not too great a difference in the ray paths of S and SKS, and also we avoid any complications associated with inner core structure.

The idea of using SKS information as a complement to S has also been used in other recent studies. Liu et al. (1996) have employed the differential times between S and SKS in a low-order spherical harmonic expansions of the threedimensional velocity structure, as a supplement to waveform data. Although there are advantages in minimising the influence of near-source and near-receiver effects, the use of differential times requires two observations at one station, which limits the number of circumstances in which it can be useful. Further, implicitly, the outer-core structure is assumed to be known so that all variation has to be accommodated by $\mathrm{S}$ variations in the mantle. Kennett et al. (1998) have also included a small number of SKS observations in their joint inversion for bulk-sound and shear wavespeed.

We will demonstrate that the inclusion of SKS data into $\mathrm{S}$ travel-time tomography enables the construction of high quality $\mathrm{S}$ wave images throughout the mantle, to provide a direct complement to the current generation of $\mathrm{P}$ wave images of three-dimensional structure. Further since we have used SKS data which is SV polarised we obtain information on the lowermost mantle which can be contrasted with previous studies which are dominated by SH wave information.

\section{S Wave Tomography}

\subsection{Data selection}

The arrival times for both S and SKS phases used in this study and the event hypocentres are taken from the global data set assembled by Engdahl et al. (1998). The entire data catalogue of the International Seismological Data Centre supplemented with more recent observations, has been relocated using a nonlinear scheme with the inclusion of the depth phases (pP, pwP) and the radially stratified ak135 model of Kennett et al. (1995) as a global reference.

The reported phases have then been re-associated using the improved locations to provide a set of travel times whose variance is significantly reduced compared with the original data catalogues. Over 35,000 events were used, recorded at a subset of over 3000 seismological stations around the globe. The careful relocation procedure removes a very major component of the variance in the original data set. We have used the $\mathrm{S}$ wave arrival-time data for distances out to $105^{\circ}$ and SKS data from $84^{\circ}$ to $105^{\circ}$.
Where cross-overs in phase times occur as at $83^{\circ}$ for $\mathrm{S}$ and SKS, particular care was taken by Engdahl et al. (1998) to ensure that the association was as effective as possible. The success of the reprocessing procedure can be well illustrated by the travel times of S and SKS used in this study which are plotted in Fig. 1(a), with a correction to surface focus. To complement the time information we display in Fig. 1(b), the ray paths for the ak135 reference model associated with $\mathrm{S}$ and SKS, which indicates the difficulty in accessing the lower mantle if attention is restricted to $S$ waves alone.

\subsection{Implementation of $S$ wave tomography}

For each of the tomographic inversions reported here we have used the same parametrisation and inversion algorithm. Following the work of van der Hilst et al. (1997) for P waves, we have employed a representation in terms of $2^{\circ} \times 2^{\circ}$ cells in latitude and longitude with 18 layers in depth. The inversion of the travel-times for three-dimensional structure has been accomplished using the LSQR algorithm as implemented by Nolet (1990) with the inclusion of gradient damping and allowance for event relocation during the extraction of structure.

We have combined the information from event clusters in a $1^{\circ} \times 1^{\circ} \times 50 \mathrm{~km}$ volume and stations in a $1^{\circ} \times 1^{\circ}$ region into a single ray path, commonly referred to as a summary ray. The use of summary rays reduces the uneven sampling of mantle structure by ray paths and the computation time required for ray-tracing, and also reduces the dimension of the matrices involved in the inversion. The datum (residual time) assigned to the summary ray was the median of all data considered for that summary ray. The number of rays that could contribute to the summary ray was not restricted.

The ray tracing in the various inversions has been carried out using the same reference model $a k 135$ as in the relocation procedure. More than one million S data from the Engdahl et al. (1998) database have been used in all, leading to a total of 306,144 summary rays for $\mathrm{S}\left(<82^{\circ}\right)$, which increase to 320,752 when the full range of $\mathrm{S}$ is included, and which is further supplemented to 347,980 when the SKS data is added. A linear system of 326,452 unknowns has to be solved for the extraction of the three-dimensional structure $(291,600$ parameters) and the event relocation parameters $(34,852)$. The structure in the outer core for the SKS observations is fixed at the ak135 velocities.

The inclusion of the 27,228 SKS data markedly improves geographical coverage in the lowermost mantle. By restricting the distance range for SKS to $84^{\circ}-105^{\circ}$ we avoid using rays with very steep ray paths which would provide little vertical resolution.

The ak135 model is well suited for use as a base model for travel-time tomography because it was constructed to match empirical travel times constructed from averages of phase times from source and station pairs across the globe. Further, both S and SKS data were explicitly included in the set of information used to construct the model (Kennett et al., 1995). The use of $a k 135$ as the reference model should therefore have the effect of minimising the potential residuals due to three-dimensional structure, so that a linearised inversion can be undertaken.

$\mathrm{S}$ travel-time residuals can be twice to four times as large as the corresponding $\mathrm{P}$ residuals, but since we deal with a 


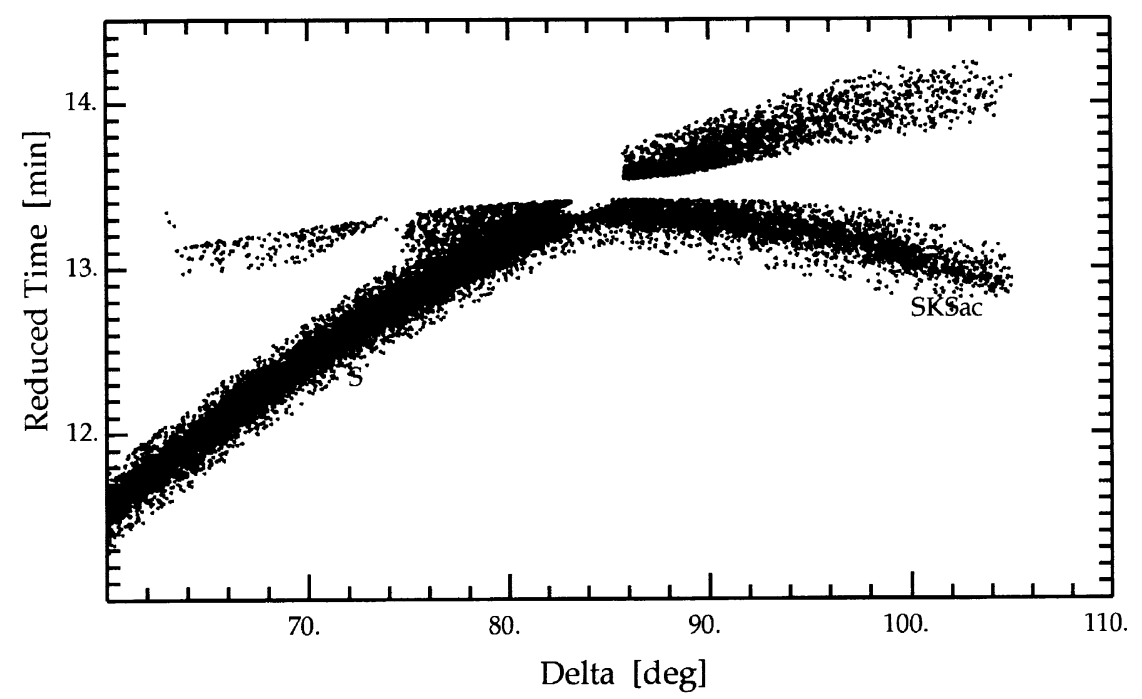

(a)

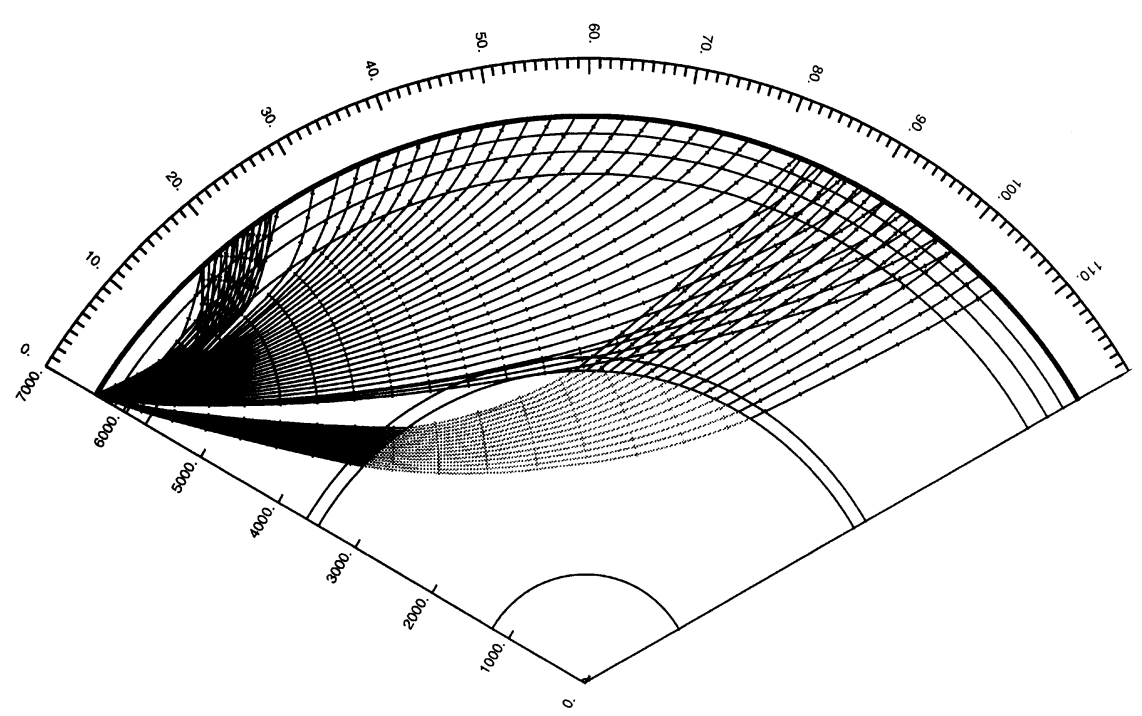

(b)

Fig. 1. (a) The travel times of S and SKS used in this study from the reprocessed data set of Engdahl et al. (1998). (b) The ray paths for S and SKS in the akl 135 model for the distance range to $105^{\circ}$ employed in the tomographic inversions.

linearised inversion scheme we have restricted the range of acceptable residuals to \pm 7.5 seconds. However, we note that as a result we may under-represent some shallower velocity structures since, for example, Sn travel-time residuals can easily reach -10.0 seconds for ray paths travelling through a fast craton. There may also be some limitation on the allowed size of features near the core-mantle boundary where large $\mathrm{S}$ residuals have been reported e.g. by Kuo and Wu (1997).

The available coverage of the mantle is restricted by the patterns of arrival time reporting from the global suite of seismic stations. Far fewer stations report $\mathrm{S}$ than $\mathrm{P}$ and there are differences in reporting patterns between regions, e.g. there is a much more limited coverage of the North American Shield than might be expected. The region of rather slow $\mathrm{S}$ velocities beneath Africa which is a major feature of many $S$ wave models e.g. Li and Romanowicz (1996) and Grand et al. (1997) is also very poorly sampled by the paths represented in the $\mathrm{S}$ dataset we have used.

During the inversion we have damped the event relocation coefficients fairly heavily in order to force the S-wave traveltime residuals to be more likely to be interpreted in terms of velocity perturbations. The variance reduction which has been achieved for each of the inversions is approximately 33 per cent which is somewhat lower than the nearly 50 per cent achieved in comparable $\mathrm{P}$ wave inversions (van der Hilst et $a l .$, 1997). This is likely to arise from the larger measurement error component in the $\mathrm{S}$ arrival-time data and the restriction on relocation.

2.2.1 Potential bias Although the model akl35 represents a good fit to the empirical travel times from which it 
was constructed, we have to accept the possibility of a discrepancy in baseline between $\mathrm{S}$ and SKS which might result in a discordance in the estimates of the residuals for each phase. However, comparison of the results for inversions with $\mathrm{S}$ alone and with the inclusion of SKS information presented in the next section suggest that any such bias is small. The results from the combined data set are in excellent agreement for those parts of the model where the $\mathrm{S}$ data achieve coverage and the geographic patterns are maintained with somewhat larger amplitude. Any significant baseline discrepancy would have the effect of displacing the influence of the SKS contribution to the three-dimensional structure with a consequent significant modification of the imaged anomalies.

\section{Comparison of S Wave Tomography Results}

\subsection{S wave inversions}

We have undertaken three separate inversions for $\mathrm{S}$ wave structure through the whole mantle with the same representation for three-dimensional velocity structure, and the same gradient and model damping parameters:

(S1) Only epicentral distances less than $82^{\circ}$ for phases associated as $\mathrm{S}$ are used. As a result we are restricted to $\mathrm{S}$ waves with a turning point above about $2100 \mathrm{~km}$ depth, and in consequence we do not have any sampling for the lowermost mantle region.

(S2) All available S phase observations are included. The impact of the cross-over between S and SKS near $83^{\circ}$ is that the number of available data in a $2^{\circ}-3^{\circ}$ distance range is markedly reduced.

$(\mathrm{S}+\mathrm{SKS})$ The full $\mathrm{S}$ phase data set is supplemented with phases associated as SKSac for distances less than $105^{\circ}$.

\subsection{Resolution tests}

Resolution tests for $\mathrm{S}$ wave structure have been carried out in two ways. Firstly, we have used a set of chequer-board tests, and secondly we have used synthetic slab models to test potential resolution of slab penetration into the lower mantle. In each case we have added Gaussian noise with a standard deviation of $1.5 \mathrm{~s}$ to the calculated travel-time residuals for the models. Figures 2 and 3 display results of the tests using the same ray coverage used to construct the S + SKS model.

In Fig. 2 we display the chequer-board pattern of $10^{\circ} \times 10^{\circ}$ cells used in odd layers of the model (Fig. 2(a)) and the recovery of this model (Figs. 2(c)-(d)). The perturbation employed is 3 per cent from the $a k 135$ reference model. In Fig. 2(b) we show the pattern recovered for layer $5(410-520 \mathrm{~km})$ using the combination of S and SKS data, which is just a little sharper than that for $\mathrm{S}$ alone. The structure is generally well resolved in the continental areas of the northern hemisphere, and in the neighbourhood of Australia. In the best resolved regions about two-thirds of the initial amplitude is recovered. In Figs. 2(c)-(d) we display the recovery of the input pattern in layer $17(2600-2750 \mathrm{~km})$ in the lowermost mantle. With just the $\mathrm{S}$ wave data (Fig. 2(c)), there is only a small region in Asia where the pattern is well recovered. However, once we include the SKS travel times there is a marked improvement in the recovery of the input pattern in both the Asian region and South American. Resolution beneath the Pacific and Africa remains weak as a consequence of the distribution of available sources and stations. There is some streaking of anomalies in the Indian and Atlantic oceans because of the lack of crossing paths in this data set.

In Fig. 3 we show a test model based on the configuration of the Tonga slab sinking into the lowermost mantle, and the recovery using linearised inversion for the $\mathrm{S}+\mathrm{SKS}$ data set which is able to image the deep structure. The S1 and $\mathrm{S} 2$ inversions are not able to resolve the deepest part of the model slab. We illustrate two different cases (i) where the model slab has penetrated into the middle of the lower mantle and (ii) where the slab material has reached the core-mantle boundary. The incorporation of the core-phase data increases the sampling in the deepest part of the mantle beneath the region as these data have a steeper take-off angle than that of the direct phases. Even so the vertical resolution at the base of the mantle is limited, but we see from Fig. 3(d) that there is little vertical smearing associated with the inclusion of SKS data. We recover about $70 \%$ of the amplitude of the heterogeneity introduced into the lower mantle.

\subsection{Tomographic images}

We present a comparison of the three-dimensional structures produced by the different inversions which allows us to examine the influence of extending the $\mathrm{S}$ data beyond the SKS cross-over (S2) and the effects arising from the inclusion of the core phase data.

In Figs. 4-6 we present the three S models i.e., S1, S2 and $\mathrm{S}+\mathrm{SKS}$ for several depth slices representing structure in the lower mantle. The S1 and S2 inversions produce visually indistinguishable results for the upper mantle and transition zone; inclusion of SKS adds some extra ray paths in the oceanic regions with very limited vertical resolution. As the depth increases differences begin to emerge between the three inversions. Figure 4 displays a slice through each model at $1300 \mathrm{~km}$ depth, at this depth the images are very close for each data set with slightly greater amplitude of anomalies with improved ray coverage. In all cases there is a clear narrow anomaly with high $\mathrm{S}$ wavespeeds extending from Indonesia across southern Eurasia which correlates very well with features seen in P wave models (van der Hilst et al., 1997), and which can be explained by subduction at the northern edge of the former Tethys ocean. There is also a narrow high wavespeed zone beneath the Americas which corresponds to that associated by Grand et al. (1997) with the subduction of the former Farallon plate, the available ray coverage does not provide as good a resolution of this feature. For the Tethys feature the contrast in structure with its surroundings is rather stronger in $\mathrm{S}$ wavespeed than the comparable high-resolution $\mathrm{P}$ tomography presented by van der Hilst et al. (1997).

At greater depths the differences between the three models start to become more significant. For example, at $2100 \mathrm{~km}$ (Fig. 5), low-velocity anomalies are apparent beneath South America in the S+SKS model (Fig. 5(c)) which do not appear in the other two models (Figs. 5(a)-(b)). The amplitude of the low wavespeed region beneath southern Africa is also enhanced. The lowered wave speeds in these regions are in accord with the recent model of Grand et al. (1997).

As would be expected, we find the largest differences be- 

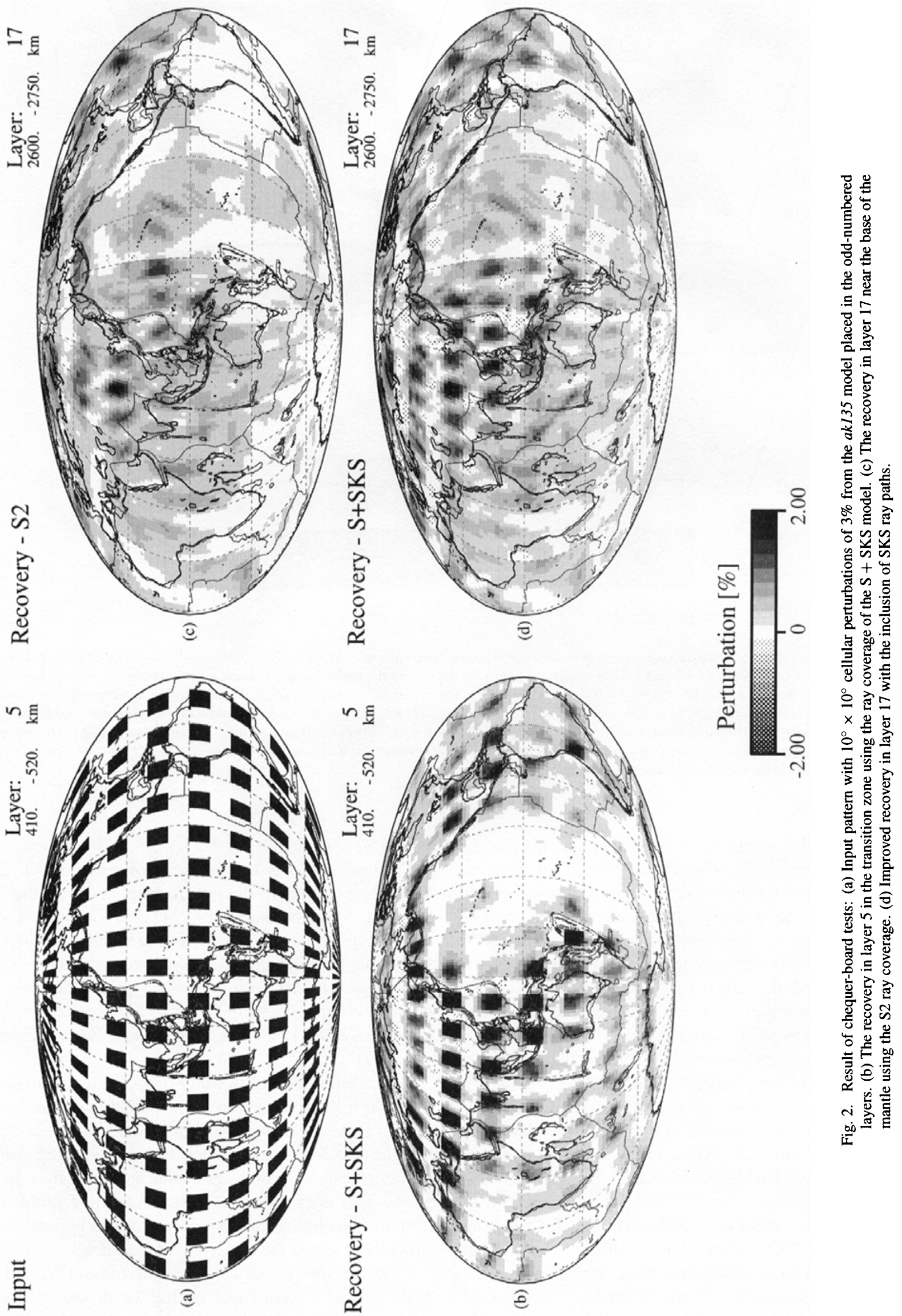

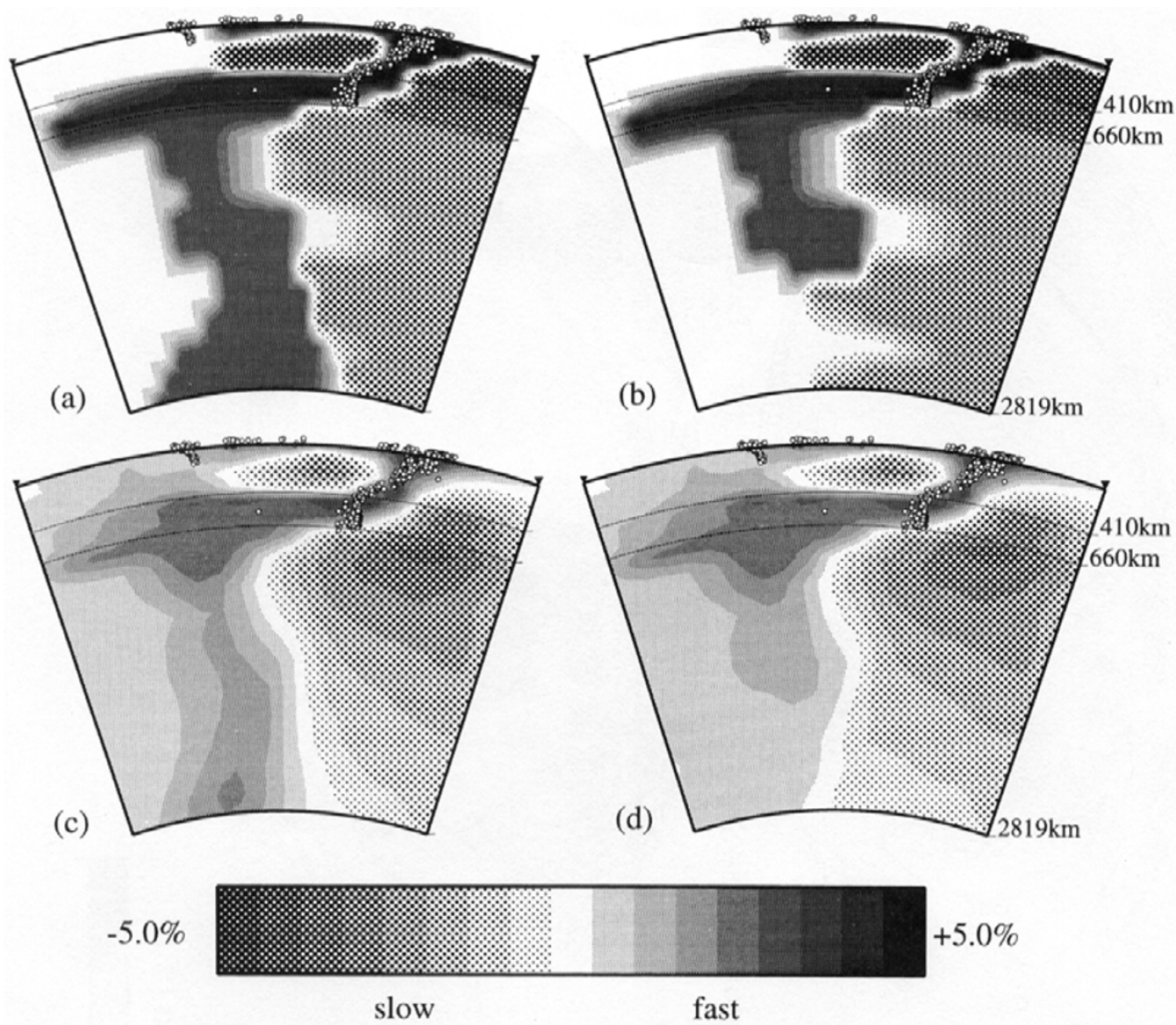

Fig. 3. (a) Model slab penetrating to the core-mantle boundary based on the Tonga region; contour scale: $-5.0 \%$ to $+5.0 \%$. Velocity perturbations in the input model are set to $+5.0 \%,+4.0 \%$ and $+3.0 \%$ relative to $a k 135$ for the slab in the upper mantle, transition zone, and lower mantle. Low-velocity anomalies outside the slab are set to $-5.0 \%,-4.0 \%$ and $-3.0 \%$ in the upper mantle, transition zone, and lower mantle, respectively. (b) Model with the slab truncated at $2000 \mathrm{~km}$ depth, to provide a check on vertical smearing. (c) Recovery of the model slab with the ray-coverage used to produce the $\mathrm{S}+\mathrm{SKS}$ model on the same contour scale. Note that the low-velocity features in the upper mantle do not smear horizontally and that the recovery of the deepest part of the model slab is reasonably good due to the inclusion of SKS phases. (d) Recovery of truncated slab model showing that high-velocity anomalies in the lower mantle do not smear vertically.

tween the different inversions in the lowermost mantle. In Fig. 6 at $2700 \mathrm{~km}$, we do not see any significant structure in the S1 inversion for the D" layer (Fig. 6(a)) since we are below the maximum turning depth of $\mathrm{S}$ waves propagating to distances less than $82^{\circ}$. The very low amplitude anomalies in Fig. 6(a) may result from seismic rays from very deep earthquakes that sample the cells in the second lowermost layer in the model parametrisation, in combination with the effects of the smoothing applied to the inversion. The inclusion of $\mathrm{S}$ data beyond the SKS cross-over in the S2 inversion (Fig. 6(b)) has begun to resolve the major features of S structure in D". However, the S + SKS inversion (Fig. 6(c)) shows that the amplitude of velocity deviations is significantly enhanced by the inclusion of the SKS phases and is closer to that suggested from waveform inversions (e.g. Li and Romanowicz, 1996).

As a further illustration of the improvements obtained from the inclusion of SKS information we display five vertical sections through the mantle across major structural features (see Fig. 7 for location) for each of the S + SKS and S2 inversions. The sections through the model derived from $\mathrm{S}+$ SKS are displayed in Fig. 8 and the corresponding sections for the S2 inversion in Fig. 9.

The first four cross-sections are chosen to represent different aspects of high $\mathrm{S}$ velocity structures in the mantle which are most likely associated with cold down-welling. The section A (Figs. 8(a) and 9(a)), across the Mediterranean region, shows that the subducted slab associated with the subducted Aegean plate penetrates into the upper part of the lower mantle in good agreement with $\mathrm{P}$ results from regional inversion (Spakman et al., 1993) and global inversion (van der Hilst et al., 1997).

Cross section B beneath Tonga (Figs. 8(b) and 9(b)) depicts a similar result to that seen in $\mathrm{P}$ regional inversion (see e.g. van der Hilst, 1995) i.e. a kink in the slab. However, there is a suggestion from the $\mathrm{S}$ models that the deflected slab may occur in the uppermost lower mantle and not in the transition zone. This does not seem to be a sampling problem and results of resolution tests using $\mathrm{P}$ and $\mathrm{S}$ data indicate that the structure is within the resolution of both data.

Cross sections C and D (Figs. 8(c)-(d) and 9(c)-(d)) beneath central America and central Japan are in excellent agreement with the results derived from P data (van der Hilst et al., 1997) and strongly support the suggestion that some 

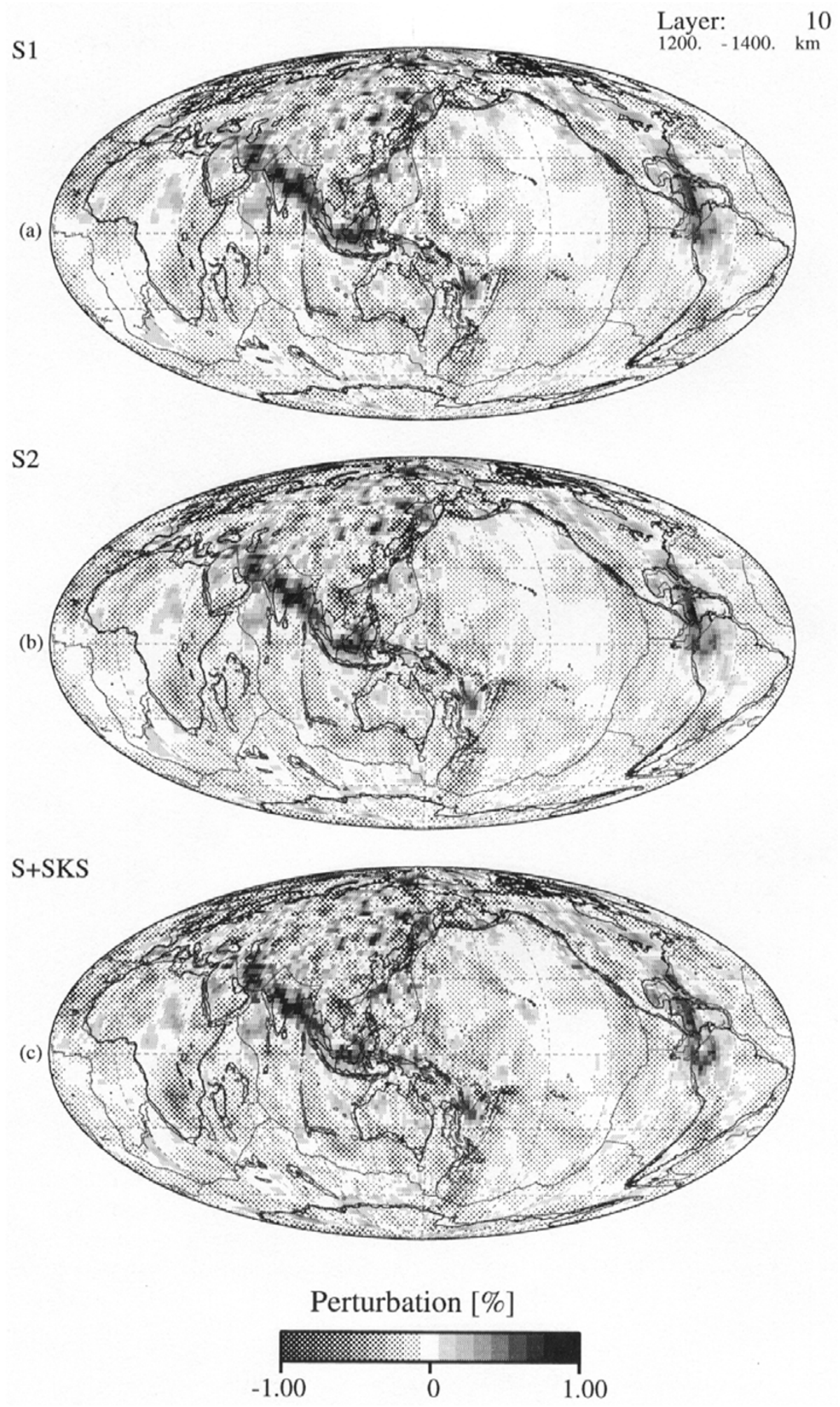

Fig. 4. S velocity anomaly maps for the three inversions at $1300 \mathrm{~km}$ depth. (a) $\mathrm{S} 1$ inversion $\left(\mathrm{S}<82^{\circ}\right)$, (b) $\mathrm{S} 2$ inversion (all $\mathrm{S}$ ), (c) $\mathrm{S}+\mathrm{SKS}$ inversion, including all $\mathrm{S}$ and SKSac to $105^{\circ}$.

slabs can sink to the lowermost mantle. The upper part of each of these sections is very similar in the two inversions but we do indeed see differences in the lowermost mantle. The high wave-speed anomaly dipping through the whole lower mantle displayed in cross section $\mathrm{C}$ may represent the slab of the Farallon plate that has subducted over the last 100
Ma or so (cf. Grand et al., 1997). The S images (Figs. 8(d) and $9(\mathrm{~d})$ ) provide confirmation of a leaky region beneath northwest Pacific which allows a down-going slab to reach the deepest part of the mantle (see van der Hilst et al., 1997, for discussion).

The final cross-section (Figs. 8(e) and 9(e)) crosses the 
S1

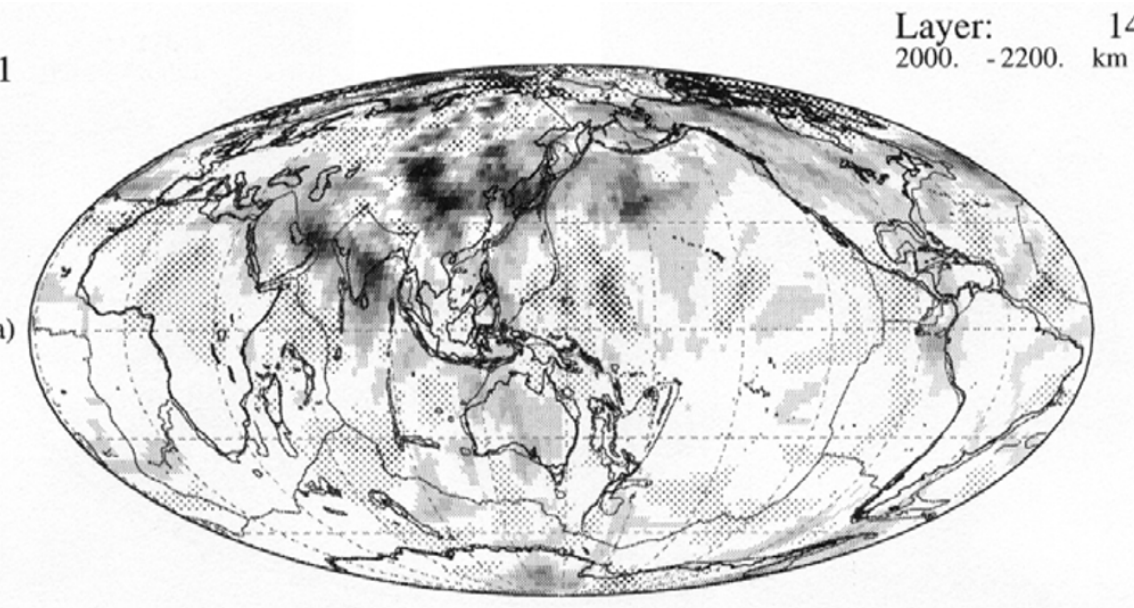

S2
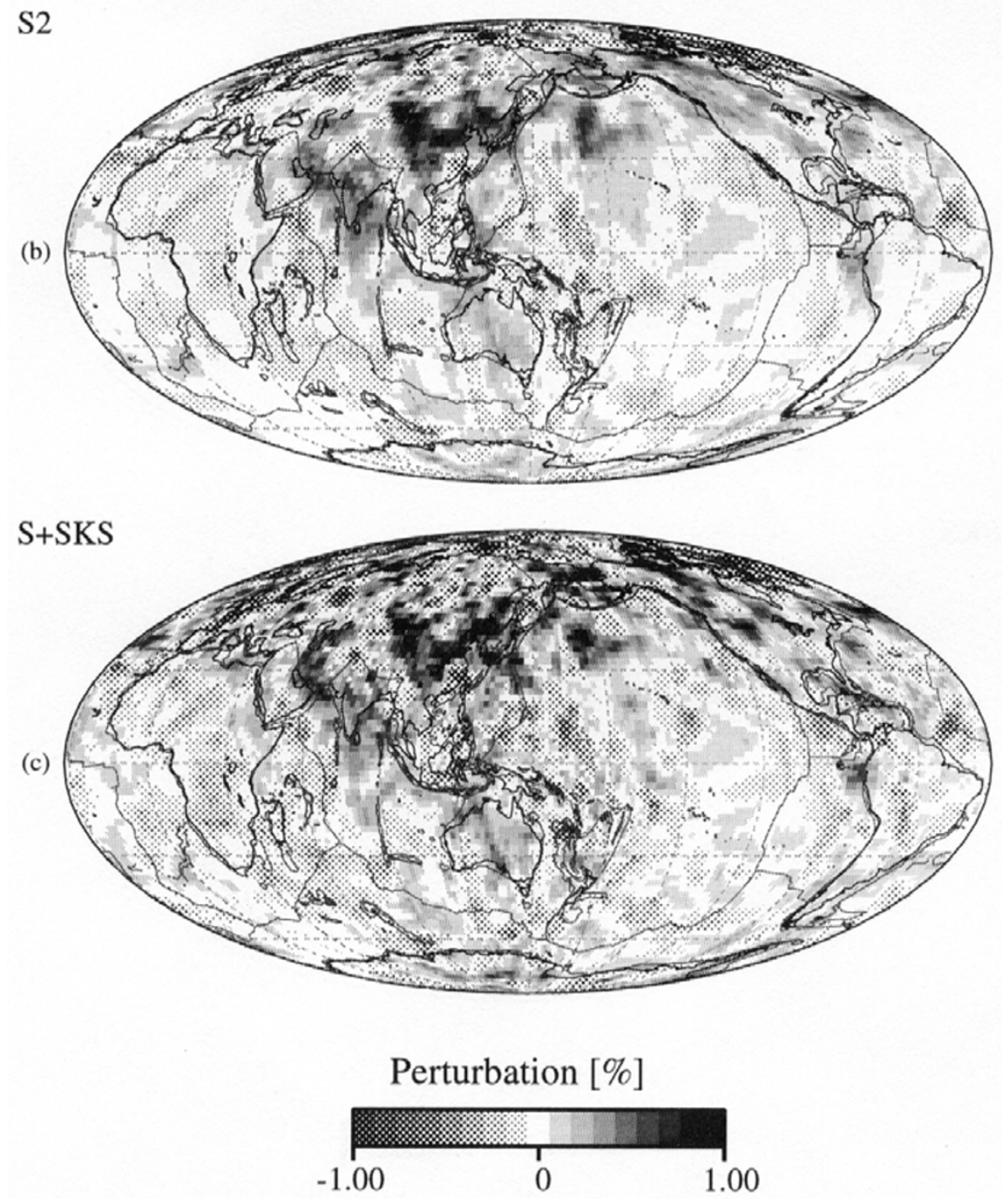

Fig. 5. S velocity anomaly maps for the three inversions at $2100 \mathrm{~km}$ depth. (a) S1 inversion, (b) S2 inversion, (c) S + SKS inversion.

region to the east of Tonga where there is a broad zone of lowered velocities (cf. Fukao et al., 1994). As in the examples with raised velocity there is good agreement between the $\mathrm{S}+\mathrm{SKS}$ and S2 inversions above $2000 \mathrm{~km}$. The inclusion of core phase information reveals a region of slightly faster $\mathrm{S}$ wavespeed in parts of the lowermost mantle (Fig. 8(e)) of which there are hints in the S2 inversion (Fig. 9(e)).

\subsection{Radial spectrum of heterogeneity}

The inclusion of the core-phase data has the effect of significantly increasing the amplitude of the deviations from the ak135 reference model in the lowermost mantle. This can be seen in Fig. 6 above, but is clearly displayed in Fig. 10 where we present a comparison of the properties of the three inversions in terms of the rms wavespeed deviations in each 

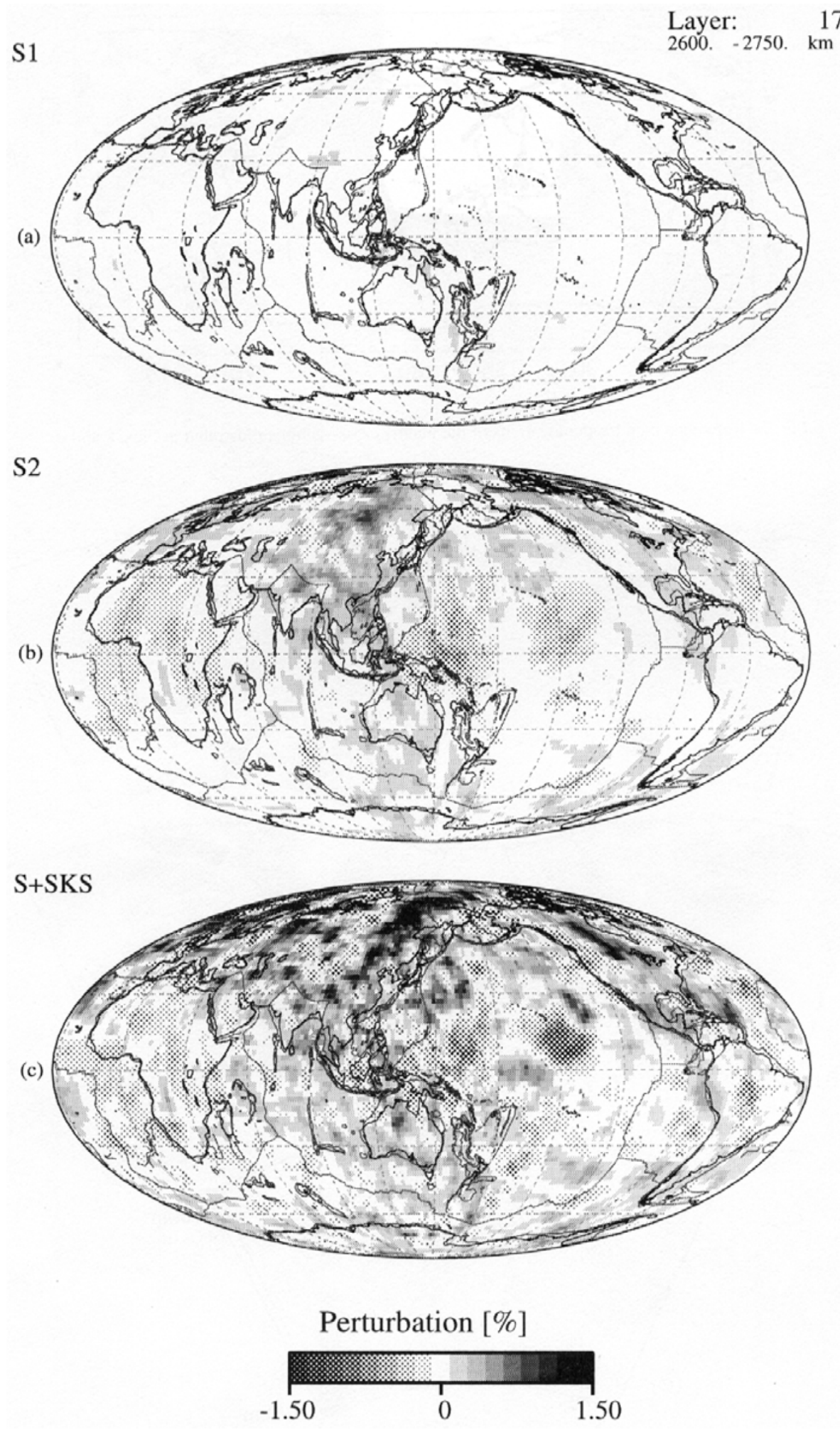

Fig. 6. S velocity anomaly maps for the three inversions at $2700 \mathrm{~km}$ depth. (a) S1 inversion, (b) S2 inversion, (c) S + SKS inversion.

layer weighted by the model sampling. The inclusion of additional $\mathrm{S}$ phase information in the $\mathrm{S} 2$ inversion introduces some amplitude into the $\mathrm{S}$ wavespeed deviations in the lowermost mantle but the heterogeneity is increased to nearly the same level as at the surface once the core phase data is also used.

In the mantle below $660 \mathrm{~km}$, where there is good crossing ray path coverage, the influence of SKS is to increase the rms variation, this arises from both enhanced geographic coverage and also reinforcement of some broadscale features. In contrast, the weighted rms deviations in the upper mantle are slightly reduced with the inclusion of the SKS information. The ray coverage in the upper mantle is rather patchy because of the limited number of reporting stations and the 


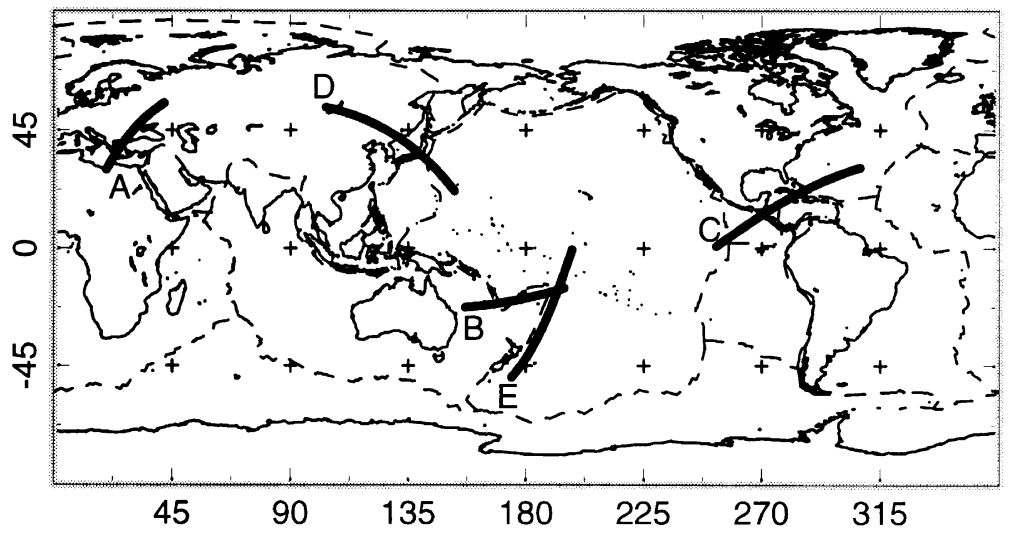

Fig. 7. Reference map for the locations of the mantle cross-sections illustrated in Figs. 8 and 9.

(a)
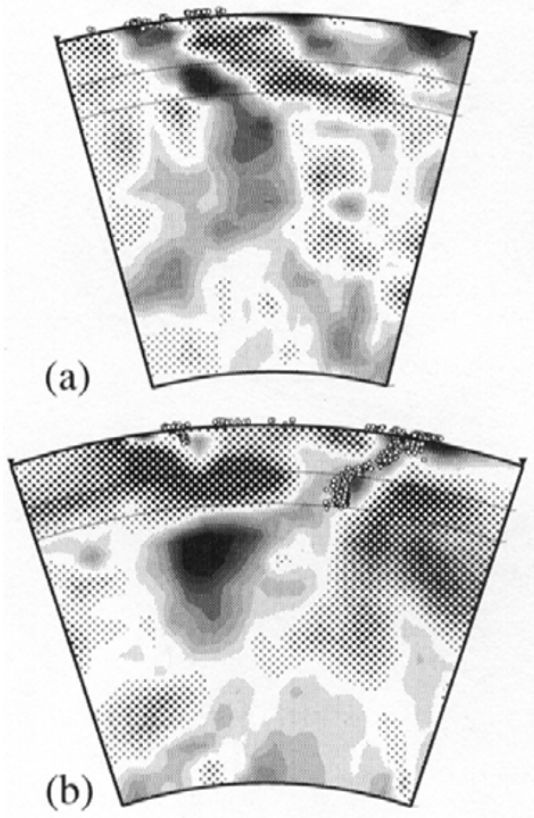

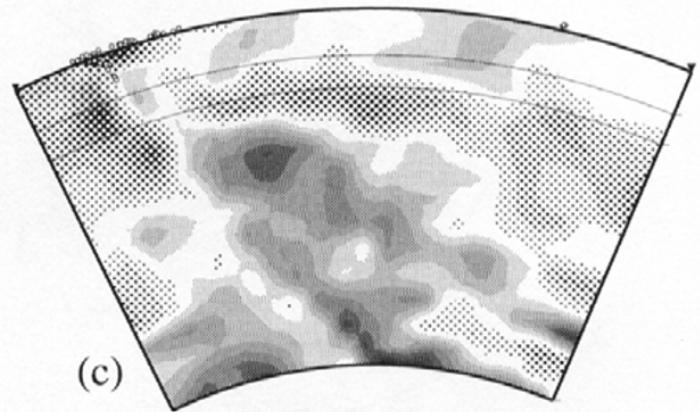

(c)

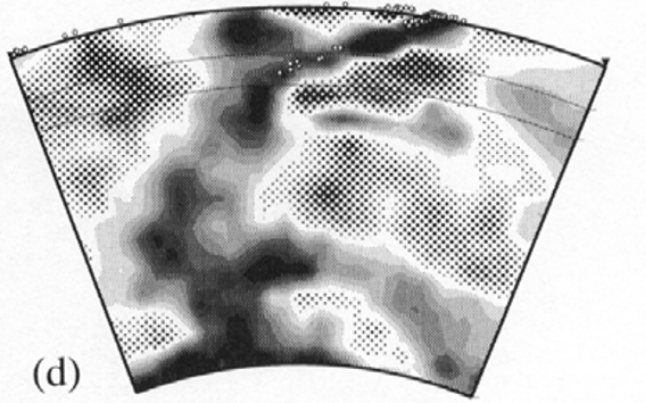

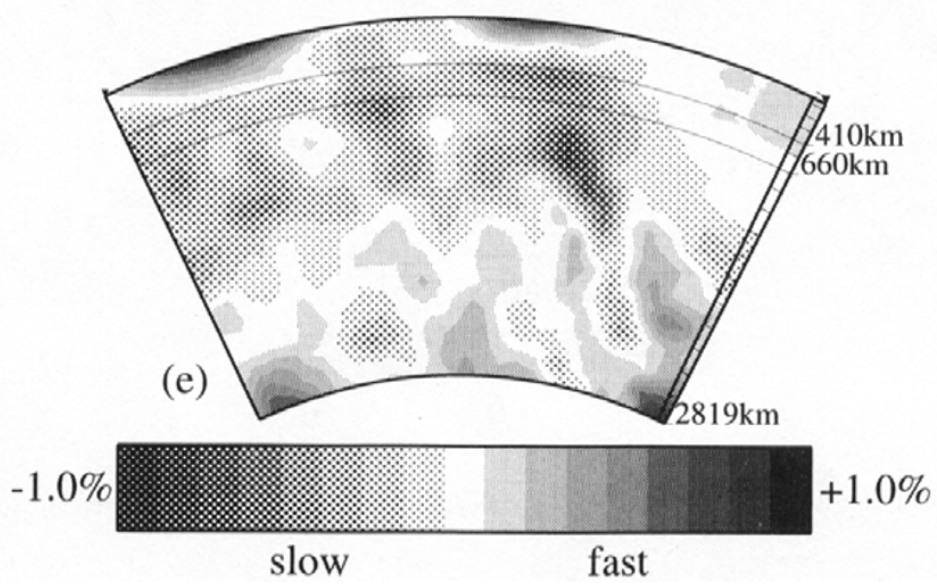

Fig. 8. Vertical mantle sections through the S wavespeed model derived from the S + SKS inversion, from the Earth's surface to the D" layer with contour scales $-1.0 \%$ to $+1.0 \%$ relative to ak135. (a) Section A, across the Aegean, is plotted from the south (left) to the north (right). (b) Section B, across Tonga, is plotted from the inner-arc in the west (left) to the outer-arc region in the east (right). (c) Section C, across central America, is plotted from southwest (left) to northeast (right). (d) Section D, across central Japan, is plotted from the inner-arc in the northwest (left) to the outer-arc region in the southeast (right). (e) Section E is plotted from south (left) to north (right). In each case, open dots superimposed in vertical cross sections depict earthquake hypocentres of magnitude $\geq 5.5$ on the Richter scale, projected from a distance of up to $50 \mathrm{~km}$ on both sides of the plane of section. The column superimposed on the image at the right-hand side of Fig. 8(e) depicts the 18 layers used in the model parametrisation. 


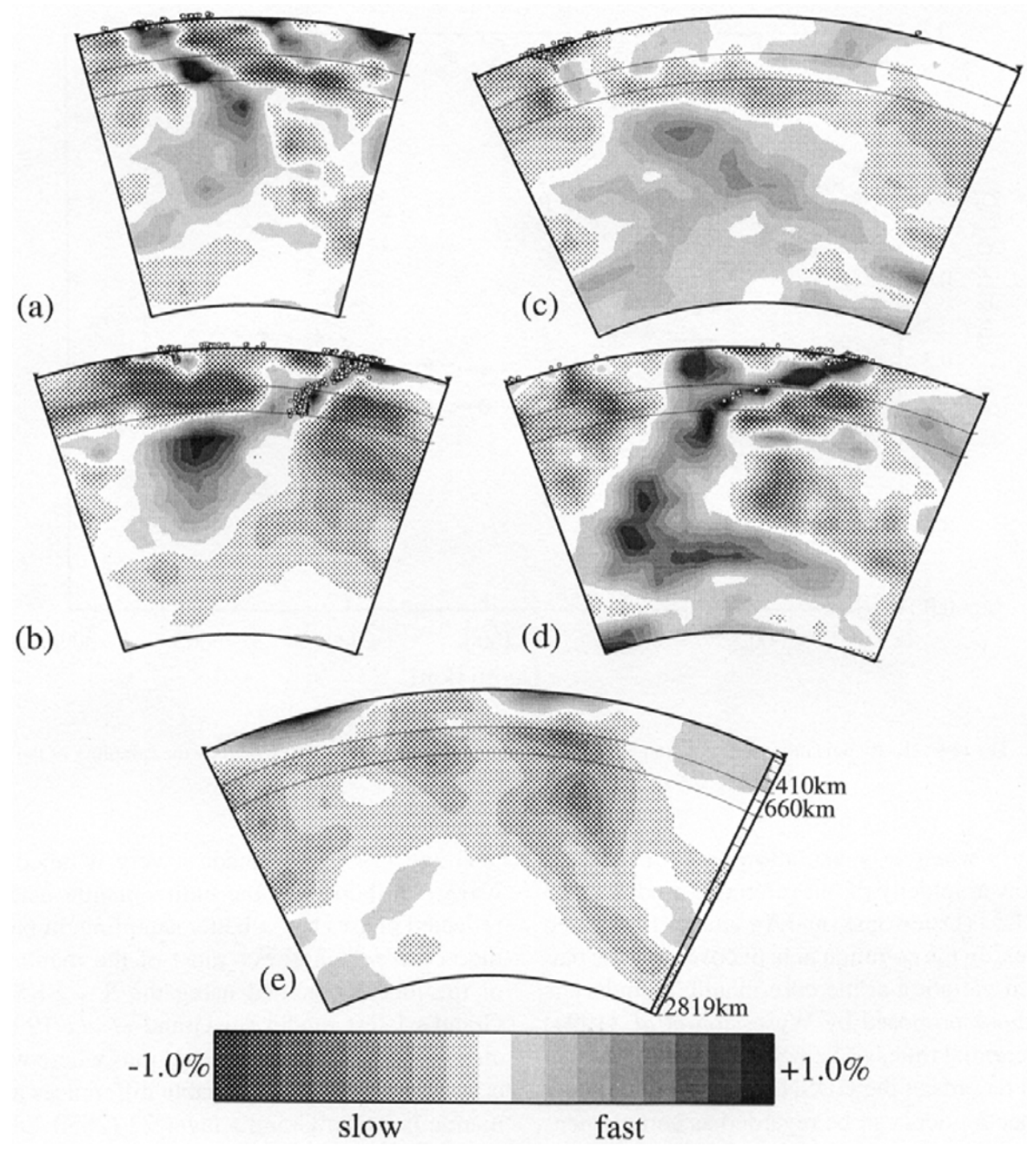

Fig. 9. Vertical mantle sections through the model derived from the $\mathrm{S} 2$ inversion with just $\mathrm{S}$ phases. The configuration of the section is precisely the same as in Fig. 8.

introduction of SKS has the effect of improving the ray path distribution with a consequent redistribution of heterogeneity away from a strong concentration of perturbation in individual cells.

The pattern of variation of $\mathrm{S}$ wavespeed heterogeneity with depth in the mantle obtained with the inclusion of the core phase information is much closer to that obtained from waveform inversions (see e.g. Li and Romanowicz, 1996) than with the use of $\mathrm{S}$ waves alone even though the amplitude of variation is somewhat lower. As we have seen in the resolution study for the slab model (Fig. 3) the level of heterogeneity we are able to recover is an underestimate of the likely situation. The clear increase in the heterogeneity level as the core-mantle boundary is approached also argues against any significant contamination by vertical smearing in the lowermost mantle with the data set we have employed.

\section{Discussion and Conclusions}

The effect of introducing SKS data has been that we have been able to extend $\mathrm{S}$ wave travel-time tomography with a $2^{\circ} \times 2^{\circ}$ cellular representation throughout the whole mantle. The resulting model has greatest resolution in the northern hemisphere and the Asian region.

From the comparison between the models derived from the S2 and S + SKS inversions in both surfaces of constant depth and vertical sections through the mantle, we can see that the incorporation of the SKSac phase times has enhanced the amplitude of velocity anomalies and has revealed additional structures arising from improved sampling. We must however retain some degree of caution, there is the possibility of some degree of vertical smearing from the steeper core phase paths and also some possible bias due to base line discrepancies between $\mathrm{S}$ and SKS.

Nevertheless the inclusion of the SKS information has enabled us to produce an $\mathrm{S}$ model for the whole mantle. The agreement between the spatial patterns of our deep mantle structure with lower resolution studies based on spherical harmonic expansion is good, e.g. Li and Romanowicz (1996) using waveform inversion, and the study using the differential times of diffracted S and SKS by Kuo and Wu 


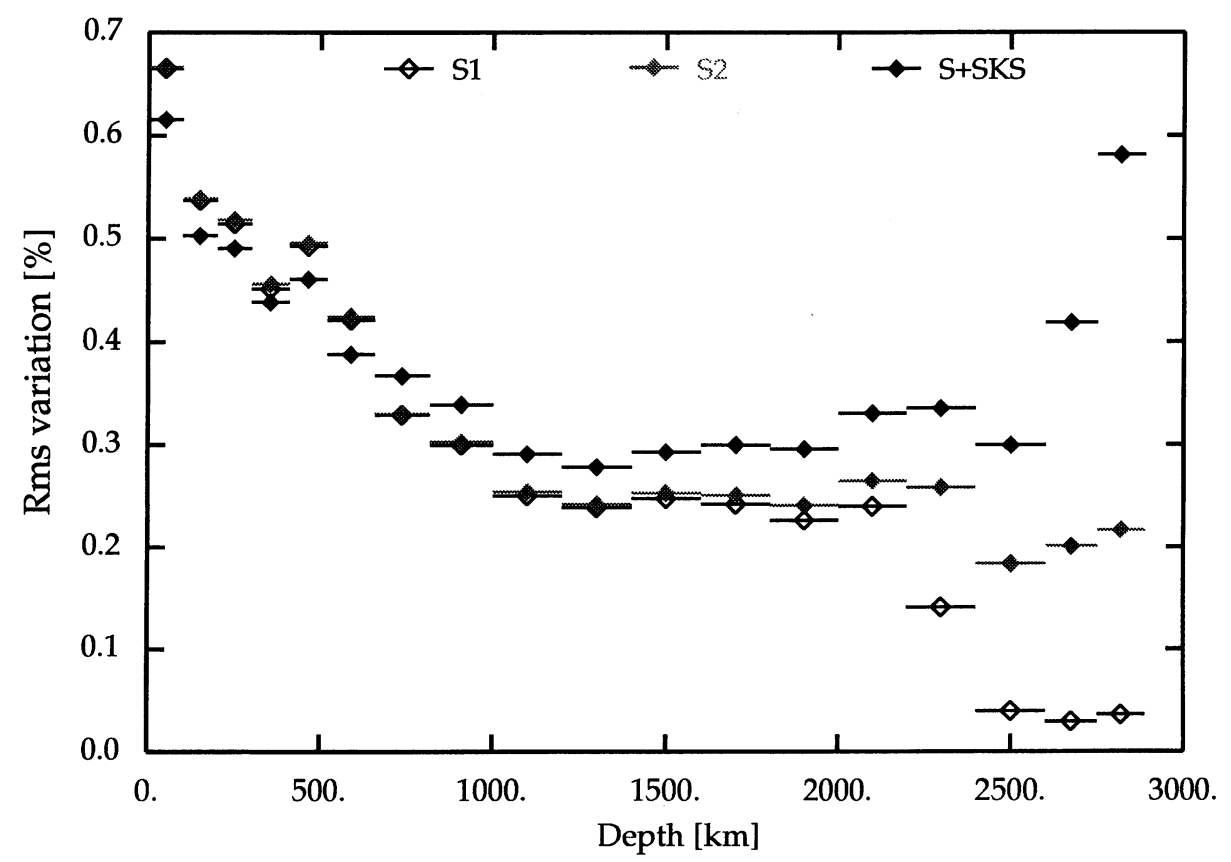

Fig. 10. The rms velocity deviations as a function of depth for each of the three models weighted by the sampling of the model.

(1997); particularly when we make allowance for the generally slightly slower velocity of our reference model $a k 135$ compared to PREM (Dziewonski and Anderson, 1981) used in the other studies. In the common area of coverage, the pattern of wavespeed variation at the core-mantle boundary is very similar to those proposed by Wysession et al. (1994) based on the differential times of ScS-S and sScS-sS derived from long period records at the GDSN network.

This $\mathrm{S}$ wavespeed model can be regarded as complementary to the three-dimensional $\mathrm{P}$ wave model presented by van der Hilst et al. (1997) using a comparable parametrisation. The ray coverage available using $\mathrm{P}$ arrival times is significantly better than we have for $\mathrm{P}$ and so it is difficult to make meaningful direct comparisons of $\mathrm{P}$ and $\mathrm{S}$ heterogeneity. This difficulty can be resolved in part by sacrificing resolution to use a restricted dataset for which $\mathrm{P}$ and $\mathrm{S}$ paths are available for the same source/station pairs. This approach has been adopted by Kennett et al. (1998) who have made a joint inversion for bulk-sound speed and shear wavespeed in the mantle using both $\mathrm{P}$ and $\mathrm{S}$ travel-time data. However, we are able to provide improved resolution of the $\mathrm{S}$ structure near the base of the mantle.

There is a good correlation between the global $\mathrm{S}$ models we present here and recent global $\mathrm{P}$ wave models derived from travel-time inversion (e.g. van der Hilst et al., 1997) down to depths of the order of $2000 \mathrm{~km}$. This result confirms the conclusions of Robertson and Woodhouse (1996) of approximate proportionality between $\mathrm{P}$ and $\mathrm{S}$ heterogeneity and helps to build further confidence in the reliability of the images. Kennett et al. (1998) from a joint inversion of $\mathrm{P}$ and $\mathrm{S}$ travel times for bulk-sound and shear wavespeed have shown that this proportionality is in large measure due to limited large-scale variation in bulk-sound speed (particularly near $2000 \mathrm{~km}$ depth).
Grand (1994) has made a very detailed study of the S wave distribution in the entire mantle using e.g. multiple reflected phases to get better sampling, in particular beneath the ocean regions. For most of the mantle, a comparison of the model obtained using the S + SKS inversion with Grand's latest model (in Grand et al., 1997) show a very close correspondence in the regions where we have good ray coverage. There are noticeable differences at the base of the mantle between Grand's layer $22(2650-2890 \mathrm{~km})$ and the results we have for layer $17(2600-2750 \mathrm{~km})$ and layer 18 $(2750-2889 \mathrm{~km})$. Both models show an increase in the size of the heterogeneity at the base of the mantle but this is much larger in Grand's model. We have seen above (Fig. 2) the loss of amplitude in our synthetic recovery tests and so this difference is likely to be a consequence of our more limited path coverage.

Much of the information we have on the structure at the base of the mantle comes from the SKS information which will represent SV wave propagation, and it is likely that much of the reported S travel times also correspond to SV waves, although the limited reports of diffracted $\mathrm{S}$ beyond $100^{\circ}$ will be dominated by SH arrivals. In the bulk of the lower mantle, the close agreement of our model with the SH wavespeed results of Grand et al. (1997) indicate that there is no need for large scale anisotropy.

In Fig. 11 we display the regions in the D" region where we have a distinct difference between the spatial patterns of our S + SKS inversion and the SH model of Grand et al. (1997), with an indication of the sense of the variations. Figure 11 represents a subjective assessment of the differences in the spatial patterns of variation between the $\mathrm{SH}$ model and the $\mathrm{SV}$ dominated model from the $\mathrm{S}+\mathrm{SKS}$ inversion. We have restricted attention to those regions for which we have adequate ray path coverage. Nevertheless, we have to be careful 


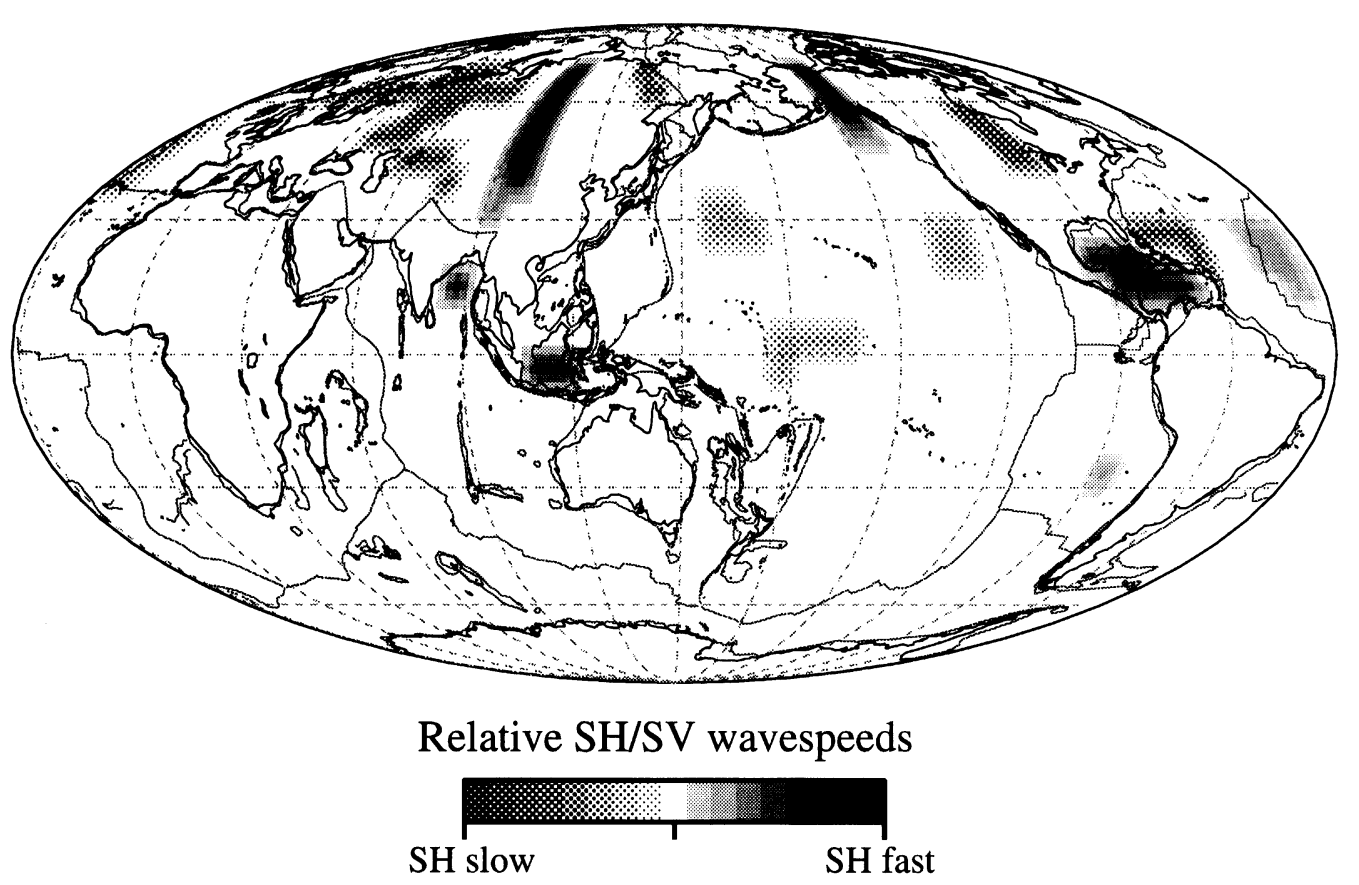

Fig. 11. The regions in the lowermost mantle where there is an indication of significant differences between the SV wavespeed derived from the S + SKS inversion [layers 17,18] and the SH wavespeed model presented by Grand et al. (1997) [layer 22]. The display shows only those portions of the model where there is adequate ray coverage from the $\mathrm{S}+\mathrm{SKS}$ dataset.

with the interpretation of these apparent differences because, as can be seen from Fig. 2(d), a number of these features fall near the edges of regions where our resolution is limited. We also have to recognise the very different sampling pattern in the work of Grand, incorporating ScS arrivals. A very striking feature of the results of Grand et al. (1997) is the very low SH wavespeeds in the lower mantle beneath Africa; our ray path coverage in this region is very sparse but we see an indication of the lowered $\mathrm{S}$ wavespeeds (see e.g. Figs. 5 and 6).

It is however interesting that a number of the differences in the spatial patterns of the SH and SV models lie in regions where there have been reports of a discontinuity above the core-mantle boundary (see e.g. Loper and Lay, 1995), notably the Caribbean and the north-south feature in central Asia. In these two regions we see that the differences between the SV and $\mathrm{SH}$ models have a doublet character with a rapid change in the relative sense of the $\mathrm{SV}$ and $\mathrm{SH}$ wavespeed perturbations.

These differences between SV and SH at the base of the mantle may correspond to the presence of shear anisotropy. There is already substantial evidence for shear wave anisotropy in this D" region (see e.g. Vinnik et al., 1995; Garnero and Lay, 1997). The resolution of the present dataset is unfortunately rather weak in the Pacific and north America which were covered in the earlier studies.

There has been recent evidence for a thin zone with greatly reduced shear wavespeed just above the core-mantle boundary particularly beneath the Pacific (Garnero and Helmberger, 1995, 1996). This zone would be penetrated by SKS waves but not refracted $S$ waves. The effect of such a thin zone of reduced wavespeed would be diminish the apparent SV wavespeed in the layer at the base of the mantle, and this may influence the low wavespeeds seen in the $\mathrm{S}+\mathrm{SKS}$ under the central Pacific. However, much of the change across the globe just above the core mantle boundary associated with the inclusion of the SKS dataset is in the form of elevated $\mathrm{S}$ wavespeeds (layer 18 is similar to layer 17 shown in Fig. 6).

All the models we have presented have been derived by a linearised inversion around the ak135 radial model. The presence of zones of large scale systematic deviation from the reference model, e.g. the very fast shield zones in the uppermost mantle, indicates that we are reaching the limits of linearised analysis. The next generation of models should include three-dimensional ray tracing on the updated models, which would be a major task even for the 300,000 or so summary rays employed in this study.

Acknowledgments. The continuing enthusiasm of Bob Engdahl for the refinement of travel-time information has been an inspiration and major benefit to this study, particularly in the reprocessing of multiple data sets to extract improved $\mathrm{S}$ information. Thanks go to Steve Grand and Wei-Ja Su for giving access to their models.

\section{References}

Dziewonski, A. M. and D. L. Anderson, Preliminary reference Earth model, Phys. Earth Planet. Inter, 25, 297-356, 1981.

Engdahl, E. R., R. D. van der Hilst, and R. Buland, Global teleseismic earthquake relocation with improved travel times and procedures for depth determination, Bull. Seism. Soc. Am., 88, 722-743, 1998.

Fukao, Y., S. Maruyama, M. Obayashi, and H. Inoue, Geologic implication of the whole mantle P-wave tomography, J. Geol. Soc. Japan, 100, 4-23, 1994.

Garnero, E. J. and D. V. Helmberger, A very slow basal layer underlying large-scale low-velocity anomalies in the lower mantle beneath the Pacific: Evidence from core phases, Phys. Earth Planet. Inter, 91, 161-176, 1995. 
Garnero, E. J. and D. V. Helmberger, Seismic detection of a thin laterally varying boundary layer at the base of the mantle beneath the central Pacific, Geophys. Res. Lett., 23, 977-980, 1996.

Garnero, E. J. and T. Lay, Lateral variations in lowermost shear wave anisotropy beneath the north Pacific and Alaska, J. Geophys. Res., 102, 8121-8135, 1997.

Grand, S. P., Mantle shear structure beneath the Americas and surrounding oceans, J. Geophys. Res., 99, 11591-11621, 1994.

Grand, S. P., R. D. van der Hilst, and S. Widiyantoro, Global seismic tomography: A snapshot of convection in the Earth, Geol. Soc. Am. Today, 7, 1-7, 1997.

Kennett, B. L. N., E. R. Engdahl, and R. Buland, Constraints on seismic velocities in the Earth from travel times, Geophys. J. Int., 122, 108-124, 1995.

Kennett, B. L. N., S. Widiyantoro, and R. D. van der Hilst, Joint seismic tomography for bulk-sound and shear wavespeed in the Earth's mantle, J. Geophys. Res., 103, 12469-12493, 1998.

Kuo, B.-Y. and K.-Y. Wu, Global shear velocity heterogeneities in the D" layer: inversion from Sd-SKS travel times, J. Geophys. Res., 102, 11775$11787,1997$.

Li, X.-D. and B. Romanowicz, Global mantle shear velocity model developed using nonlinear asymptotic coupling theory, J. Geophys. Res., 101, 22245-22272, 1996.

Liu, X.-F., W.-J. Su, and A. M. Dziewonski, Improved resolution of the lowermost shear velocity structure using SKS-S data, EOS, Trans. $A G U$, 75(16), Spring Meeting Suppl., 232, 1996.

Loper, D. and T. Lay, The core-mantle boundary region, J. Geophys. Res., 100, 6397-6420, 1995

Masters, G., S. Johnson, G. Laske, and H. Bolton, A shear-velocity model of the mantle, Phil. Trans. R. Soc. Lond., 354A, 1385-1411, 1996.

Morelli, A. and A. M. Dziewonski, Body wave travel times and a spherically symmetric P- and S-wave velocity model, Geophys. J. Int., 112, 178-194 1993.

Nolet, G., Seismic wave propagation and seismic tomography, in Seismic Tomography, edited by G. Nolet, pp. 1-23, D. Reidel, Dordrecht, 1990.

Robertson, G. S. and J. H. Woodhouse, Evidence for the proportionality of $\mathrm{P}$ and $\mathrm{S}$ heterogeneity in the mantle, Geophys. J. Int., 123, 85-116, 1995.

Robertson, G. S. and J. H. Woodhouse, Ratio of relative S to P velocity heterogeneities in the lower mantle, J. Geophys. Res., 101, 20041-20052, 1996.

Spakman, W., S. van der Lee, and R. D. van der Hilst, Travel-time tomography of the European-Mediterranean mantle down to $1400 \mathrm{~km}$, Phys. Earth Planet. Inter., 79, 3-74, 1993.

van der Hilst, R. D., Complex morphology of subducted lithosphere in the mantle beneath the Tonga trench, Nature, 374, 154-157, 1995.

van der Hilst, R. D., S. Widiyantoro, and E. R. Engdahl, Evidence for deep mantle circulation from global tomography, Nature, 386, 578-584, 1997.

Vasco, D. W., L. R. Johnson, R. J. Pulliam, and P. S. Earle, Robust inversion of IASP91 travel-time residuals for mantle $\mathrm{P}$ and $\mathrm{S}$ structure, earthquake mislocations, and station corrections, J. Geophys. Res., 99, 13727-13755, 1994.

Vinnik, L. V., B. Romanowicz, Y. Le Stunff, and L. Makeyeva, Seismic anisotropy of the D" layer, Geophys. Res. Lett., 22, 1657-1660, 1995.

Wysession, M. E., L. Bartkó, and J. B. Wilson, Mapping the lowermost mantle using core-reflected shear waves, J. Geophys. Res., 99, 1366713684, 1994.

S. Widiyantoro (e-mail: sriwid@eri.u-tokyo.ac.jp), B. L. N. Kennett (e-mail: brian@rses.anu.edu.au), and R. D. van der Hilst (e-mail: hilst@ mit.edu) 
S. WIDIYANTORO et al.: EXTENDING SHEAR-WAVE TOMOGRAPHY USING S AND SKS DATA

Fig. 2. Result of chequer-board tests: (a) Input pattern with $10^{\circ} \times 10^{\circ}$ cellular perturbations of $3 \%$ from the $a k 135$ model placed in the odd-numbered layers. (b) The recovery in layer 5 in the transition zone using the ray coverage of the S + SKS model. (c) The recovery in layer 17 near the base of the mantle using the S2 ray coverage. (d) Improved recovery in layer 17 with the inclusion of SKS ray paths. 